\title{
Linoleic acid-derived lipid mediators increase in a female-dominated subphenotype of COPD
}

\author{
David Balgoma ${ }^{1,2}$, Mingxing Yang ${ }^{3}$, Marcus Sjödin ${ }^{1}$, Stuart Snowden ${ }^{1}$, \\ Reza Karimi ${ }^{3}$, Bettina Levänen ${ }^{3}$, Heta Merikallio ${ }^{3,4}$, Riitta Kaarteenaho ${ }^{4}$, \\ Lena Palmberg ${ }^{5}$, Kjell Larsson ${ }^{5}$, David J. Erle ${ }^{6}$, Sven-Erik Dahlén ${ }^{2}$, \\ Barbro Dahlén 7 , C. Magnus Sköld ${ }^{3}$, Åsa M. Wheelock ${ }^{3,8}$ and Craig E. Wheelock ${ }^{1,8}$ \\ Affiliations: 'Division of Physiological Chemistry 2, Department of Medical Biochemistry and Biophysics, \\ Karolinska Institutet, Stockholm, Sweden. ${ }^{2}$ Institute of Environmental Medicine, Karolinska Institutet, \\ Stockholm, Sweden. ${ }^{3}$ Respiratory Medicine Unit, Dept of Medicine Solna and Center for Molecular Medicine, \\ Karolinska Institutet, Stockholm, Sweden. ${ }^{4}$ Respiratory Unit, Medical Research Center Oulu, Oulu University/ \\ UniversityHospital, Medicine and Clinical Research, Pulmonary Division, University of Eastern Finland \& \\ Division of Respiratory Medicine, Kuopio University Hospital, Kuopio, Finland. ${ }^{5}$ Lung and Airway Research \\ Unit, Institute of Environmental Medicine, Karolinska Institutet, Stockholm, Sweden. ${ }^{6}$ Division of Pulmonary \\ and Critical Care, Department of Medicine and Lung Biology Center, University of California San Francisco, \\ San Francisco, USA. ${ }^{7}$ Department of Respiratory Medicine and Allergy, Karolinska University Hospital \\ Huddinge, Stockholm, Sweden. ${ }^{8}$ Both authors contributed equally.
}

Correspondence: Craig E. Wheelock, Division of Physiological Chemistry 2, Department of Medical Biochemistry and Biophysics, Karolinska Institutet, 17177 Stockholm, Sweden. E-mail: craig.wheelockaki.se

ABSTRACT Chronic obstructive pulmonary disease (COPD) is a leading cause of mortality; however, the role of inflammatory mediators in its pathobiology remains unclear. The aim of this study was to investigate the influence of gender in COPD on lipid mediator levels.

Bronchoalveolar lavage fluid (BALF) and serum were obtained from healthy never-smokers, smokers and COPD patients (Global Initiative for Chronic Obstructive Lung Disease stage I-II/A-B) (n=114). 94 lipid mediators derived from the cytochrome-P450, lipoxygenase, and cyclooxygenase pathways were analysed by liquid chromatography-mass spectrometry.

Multivariate modelling identified a 9-lipid panel in BALF that classified female smokers with COPD from healthy female smokers $\left(p=6 \times 10^{-6}\right)$. No differences were observed for the corresponding male population $(\mathrm{p}=1.0)$. These findings were replicated in an independent cohort with $92 \%$ accuracy $(\mathrm{p}=0.005)$. The strongest drivers were the cytochrome $\mathrm{P} 450$-derived epoxide products of linoleic acid (leukotoxins) and their corresponding soluble epoxide hydrolase (sEH)-derived products (leukotoxindiols). These species correlated with lung function $(\mathrm{r}=0.87 ; \mathrm{p}=0.0009)$ and mRNA levels of enzymes putatively involved in their biosynthesis $(\mathrm{r}=0.96 ; \mathrm{p}=0.003)$. Leukotoxin levels correlated with goblet cell abundance $(\mathrm{r}=0.72 ; \mathrm{p}=0.028)$.

These findings suggest a mechanism by which goblet cell-associated cytochrome-P450 and sEH activity produce elevated leukotoxin-diol levels, which play a putative role in the clinical manifestations of COPD in a female-dominated disease sub-phenotype.

@ERSpublications

Linoleic acid-derived lipids in BALF may indicate the transition from healthy smoker to COPD in a female subphenotype http://ow.ly/XowqN

This article has supplementary material available from erj.ersjournals.com

Received: July 062015 | Accepted after revision: Jan 13 2016 | First published online: March 102016

Clinical trial: This study is registered at ClinicalTrials.gov with identifier number NCT02627872.

Conflict of interest: Disclosures can be found alongside the online version of this article at erj.ersjournals.com

Copyright @ERS 2016 


\section{Introduction}

Chronic obstructive pulmonary disease (COPD) is a leading cause of morbidity and mortality worldwide [1]. It is characterised by persistent and non-reversible airflow limitation associated with airway inflammation and remodelling [2]. The primary risk factor for COPD in western countries is smoking, with $15-44 \%$ of the population developing disease [1]. COPD evidences gender dependency with higher mortality in women, even after correction for smoking [3, 4]. Smoking also results in greater impairment in lung function in women $[5,6]$, especially post-menopause [7].

COPD is a heterogeneous disease with a variety of inflammatory pathways involved in disease pathophysiology [8]. The current study was designed to investigate the potential role of lipid mediators in the heterogeneity of COPD, with particular focus on examining gender associated differences. Female gender has been suggested as a risk factor in susceptibility to the lung-damaging effects of cigarette smoke, and both smokers and nonsmokers with COPD are more likely to be female $[4,6,9]$. Lipid mediators are known to play an important role in the inflammatory cascade in respiratory disease, including COPD (for review, see [10]) [11-13]. The most well studied of these lipid mediators are the eicosanoids, which are synthesised from arachidonic acid via three biosynthetic pathways (figure E1 in the supplementary material): cyclooxygenase (COX), lipoxygenase (LOX), cytochrome P450 (CYP), as well as via non-enzymatic oxidation. However, there are numerous lipid species synthesised from other polyunsaturated fatty acids that exhibit biological activity. For example, the linoleic acid-derived leukotoxins (epoxy-octadecenoic acids (EpOMEs)) and leukotoxin-diols (dihydroxy-octadecenoic acids (DiHOMEs)) (figure 1) have been reported to play a role in acute respiratory distress syndrome (ARDS) [14-16] and exert toxicity to alveolar epithelial cells [17]. Accordingly, there is interest in further investigating these lipid mediator pathways in the pathobiology of COPD.

Methods

Subjects and study design

This study examined subjects from the Karolinska COSMIC (Clinical \& Systems Medicine Investigations of Smoking-related COPD) cohort at the Karolinska University Hospital (ClinicalTrials.gov identifier NCT02627872). The COSMIC study is a three-group cross-sectional study in which each group was stratified by sex with the aim of investigating the differentiation between the genders in early stage COPD and integrating several aspects of COPD and smoking through the use of imaging, transcriptomics, proteomics, metabolomics, and lymphocyte profiling in the context of clinical phenotypes [18-21]. A total of 40 never-smokers, 40 smokers with normal lung function (hereafter referred to as "smokers"), and 38 patients with COPD were recruited with the intent to collect peripheral blood and bronchoalveolar lavage (BAL). Of the 118 recruited individuals, three individuals with COPD and one never-smoker did not undergo BAL because of clinical constraints and were therefore excluded from the current analysis. The study was accordingly performed on 114 subjects from the Karolinska COSMIC cohort (table 1) matched for age and sex from the groups of healthy never-smokers, smokers with normal lung function and COPD patients with mild-to-moderate disease (Global Initiative for Chronic Obstructive Lung Disease (GOLD) stage I-II/A-B; forced expiratory volume in $1 \mathrm{~s}$ (FEV1) 51-97\%; FEV1/forced expiratory volume (FVC) $<70)[18,20]$.

Study participants were recruited from individuals performing spirometry during "World Spirometry Day", through advertisements in the daily press and via primary care centres. The majority of the individuals with COPD were smokers who were found to have an obstructive spirometry upon screening. Participants had no history of allergy or asthma, did not use inhaled or oral corticosteroids and had no exacerbations for at least 3 months prior to study inclusion. In vitro screenings for the presence of specific immunoglobulin (Ig)E antibodies (Phadiatop; ImmunoCAP, Phadia, Uppsala, Sweden) were negative. Reversibility was tested after inhalation of two doses of $0.25 \mathrm{mg}$ terbutaline (Bricanyl Turbuhaler; AstraZeneca, London, UK). Medications (including oral contraceptives, oestrogen replacement and nonsteroidal anti-inflammatory drugs or other potential lipid mediator-modifying drugs) were recorded by means of a questionnaire. Lung function parameters were calculated as post-bronchodilator percentage

Support statement: This study was funded by the Swedish Heart-Lung Foundation, Swedish Foundation for Strategic Research (SSF), VINNOVA (VINN-MER), EU FP6 Marie Curie, Karolinska Institutet, COST BM1201, VINNOVA (CiDAT), AFA Insurance, the King Oscar II Jubilee Foundation, the King Gustaf V and Queen Victoria's Freemasons Foundation, the Finnish Anti-Tuberculosis Association Foundation, the Swedish Research Council, the regional agreement on medical training and clinical research (ALF) between Stockholm County Council and Karolinska Institutet, the Centre for Allergy Research, and the Karolinska Institutet and AstraZeneca Joint Research Program in Translational Science. D.B. and M.S. received support from U-BIOPRED, the Innovative Medicines Initiative Joint Undertaking under grant agreement no. 115010, resources of which are composed of financial contribution from the European Union's Seventh Framework Programme (FP7/2007-2013) and EFPIA companies' in kind contribution. Funding information for this manuscript has been deposited with FundRef. 


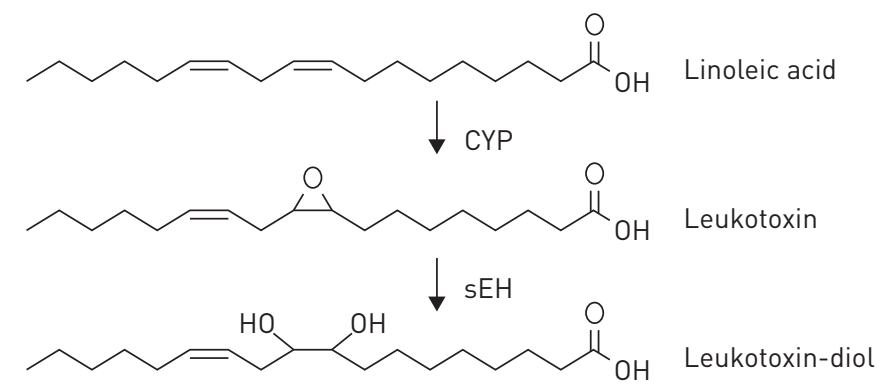

FIGURE 1 Biosynthetic pathway for production of leukotoxin and leukotoxin-diol from linoleic acid. Linoleic acid is converted by cytochrome P450 (CYP) activity to either 9(10)-epoxy-12Z-octadecenoic acid (leukotoxin, coronaric acid, 9[10]-EpOME) or the regioisomer 12(13)-epoxy-12Z-octadecenoic acid (isoleukotoxin, vernolic acid, 12[13]-EpOME), which can be hydrolysed to the corresponding vicinal diols (leukotoxin-diol, 9,10-DiHOME and isoleukotoxin-diol, 12,13-DiHOME, respectively) by soluble epoxide hydrolase (sEH) activity.

predicted using the European Community of Coal and Steel (ECCS) normal values. COPD patients and smokers were matched in terms of smoking history (>10 pack-years) and current smoking habits ( $>10$ cigarettes day ${ }^{-1}$ in the past 6 months). The COPD group consisted of both current smokers and ex-smokers ( $\geqslant 2$ years since smoking cessation). Because the current study focused on mild-to-moderate COPD, there was an overlap between post-bronchodilatory $\mathrm{FEV}_{1} \%$ pred (but not with the FEV1/FVC ratio) between healthy individuals and participants with COPD; however, in all cases, the current guidelines on COPD were employed when defining study inclusion criteria.

The validation cohort consisted of two gender groups recruited to the same study [22]. Exclusion criteria included a history of asthma or other pulmonary or allergic disease. The participants were clinically stable at the time of the study and were excluded if they had experienced an airway infection during the 14 days prior to study initiation. All medication was withheld $48 \mathrm{~h}$ prior to clinical visit and BAL. The female group consisted of seven female current smokers (49-61 years; body-mass index (BMI) 20.5-32.6) with normal lung function and six female current smokers with COPD (48-73 years; BMI 20.0-27.7; GOLD stage II-III; FEV1 44-68\%; FEV1/FVC $<70)$ with arterial oxygen saturation $\left(\mathrm{SaO}_{2}\right)>90 \%$. The male group consisted of nine male current smokers (41-66 years; BMI 21.7-31.4) with normal lung function and five male current smokers with COPD (53-70 years; BMI 17.3-24.6; GOLD stage I-III; FEV1 37-99\%; FEV1/ FVC<70) with $\mathrm{SaO}_{2}>90 \%$ [22].

Both studies were approved by the Stockholm Regional Ethical Board (COSMIC cohort: No. 2006/959-31/ 1; validation cohort: No. 2005/733-31/1-4) and participants provided their informed written consent.

\section{Sample collection and preparation}

Bronchoalveolar lavage was performed and airway epithelial brushings collected as previously described (see online supplement) $[18,23]$. In order to investigate cells involved in first line host defense from both large and small airways, cells from epithelial brushings and bronchoalveolar lavage were chosen. Samples were aliquoted and stored at $-80^{\circ} \mathrm{C}$ until analysis. Blood was drawn between 7-9 AM from fasting individuals by venipuncture and allowed to stand at room temperature for at least 30 min before centrifugation at $1695 \times \mathrm{g}$ for $10 \mathrm{~min}$ at room temperature, and stored at $-80^{\circ} \mathrm{C}$ until use. High-sensitivity C-reactive protein (CRP), platelet and white blood cell counts were measured according to standard methods at the Department of Clinical Chemistry, Karolinska University Hospital. Cytospins prepared from bronchial brushings were fixed with cold acetone, and stained with the alcian blue-periodic acid Schiff (AB-PAS) technique according to standard methods. Differential count of BAL cells was performed by means of Giemsa-Grunwald staining of cytospins.

\section{Lipid mediator analyses}

A liquid chromatography-mass spectrometry (LC-MS/MS) method was developed to quantify the reported lipid mediators. The complete method is described in the online supplement, with lipid mediator nomenclature provided in table E1. Briefly, $3.3 \mathrm{~mL}$ of BAL fluid (BALF) were mixed with $10 \mu \mathrm{L}$ of internal standards in concentrations according to table E2 and loaded onto Waters Oasis HLB solid phase extraction (SPE) cartridges (Waters, Milford, MA, USA). SPE cartridges were air-dried, and lipid mediators eluted with organic solvent, evaporated under vacuum and reconstituted in $100 \mu \mathrm{L}$ of methanol. Following spin filtering, $7.5 \mu \mathrm{L}$ were injected onto an Acquity ultra performance liquid chromatography with a BEH C18 column $(2.1 \times 150 \mathrm{~mm}, 1.7 \mu \mathrm{m}$; Waters $)$ and analysed on a Waters Xevo TQ-MS in negative mode. The calibration levels and method parameters of all analysed compounds are provided in 
TABLE 1 Clinical characteristics of study subjects

\begin{tabular}{|c|c|c|c|c|c|c|c|c|}
\hline & \multicolumn{2}{|c|}{ Healthy } & \multicolumn{2}{|c|}{ Smokers } & \multicolumn{2}{|c|}{ COPD } & \multicolumn{2}{|c|}{ COPD ExS } \\
\hline Group size $\mathbf{n}$ & 19 & 20 & 20 & 20 & 14 & 11 & 4 & 6 \\
\hline \multicolumn{9}{|l|}{ GOLD stage } \\
\hline 1 & NA & NA & NA & NA & 7 & 6 & 2 & 4 \\
\hline II & NA & NA & NA & NA & 7 & 5 & 2 & 2 \\
\hline$A$ & NA & NA & NA & NA & 10 & 8 & 3 & 4 \\
\hline BMI $\mathrm{kg} \cdot \mathrm{m}^{-2}$ & $27 \pm 5$ & $26 \pm 4$ & $24 \pm 4$ & $24 \pm 2$ & $24 \pm 4$ & $24 \pm 4$ & $27 \pm 4$ & $28 \pm 5$ \\
\hline $\mathrm{CRP} \mathrm{mg} \cdot \mathrm{L}^{-1}$ & $1.3 \pm 0.91$ & $1.9 \pm 1.7$ & $4.4 \pm 3.2$ & $2.6 \pm 1.1$ & $1.2 \pm 0.88$ & $1.9 \pm 1.9$ & $1.7 \pm 2.2$ & $2.7 \pm 2.4$ \\
\hline Recovery BAL \% & $62 \pm 14$ & $67 \pm 8$ & $55 \pm 10$ & $63 \pm 9$ & $47 \pm 12$ & $40 \pm 18$ & $42 \pm 12$ & $55 \pm 10$ \\
\hline Smoking history pack-years & 0 & 0 & $35 \pm 15$ & $35 \pm 11$ & $44 \pm 11$ & $39 \pm 9$ & $28 \pm 8$ & $28 \pm 9$ \\
\hline FEV $1 \%$ & $118 \pm 14$ & $120 \pm 12$ & $109 \pm 11$ & $109 \pm 13$ & $77 \pm 9$ & $82 \pm 13$ & $76 \pm 19$ & $81 \pm 11$ \\
\hline FEV $1 /$ VC \% & $79 \pm 9$ & $81 \pm 6$ & $76 \pm 5$ & $76 \pm 7$ & $59 \pm 9$ & $60 \pm 9$ & $54 \pm 17$ & $60 \pm 7$ \\
\hline BAL cell concentration $\times 10^{6} \cdot \mathrm{L}^{-1}$ & $123 \pm 55.9$ & $119 \pm 44.4$ & $551 \pm 267$ & $561 \pm 258$ & $528 \pm 267$ & $447 \pm 244$ & $107 \pm 19.9$ & $125 \pm 87.2$ \\
\hline BAL viable cells $\%$ & $93.4 \pm 3.85$ & $93.2 \pm 4.77$ & $91.6 \pm 5.58$ & $91.8 \pm 3.32$ & $91.4 \pm 3.22$ & $92.2 \pm 3.97$ & $94.3 \pm 2.06$ & $91.4 \pm 5.19$ \\
\hline BAL macrophages $\times 10^{6} \cdot \mathrm{L}^{-1}$ & $105 \pm 46.3$ & $102 \pm 35.0$ & $529 \pm 258$ & $541 \pm 248$ & $505 \pm 263$ & $427 \pm 237$ & $93.0 \pm 15.4$ & $106 \pm 81.4$ \\
\hline BAL lymphocytes $\times 10^{6} \cdot \mathrm{L}^{-1}$ & $16.9 \pm 20.1$ & $14.3 \pm 15.4$ & $15.4 \pm 13.7$ & $12.8 \pm 9.02$ & $13.0 \pm 10.4$ & $13.4 \pm 7.21$ & $10.1 \pm 7.56$ & $16.9 \pm 8.29$ \\
\hline BAL neutrophils $\times 10^{6} \cdot \mathrm{L}^{-1}$ & $1.57 \pm 1.30$ & $2.21 \pm 1.84$ & $4.44 \pm 3.04$ & $5.33 \pm 6.98$ & $4.71 \pm 3.23$ & $5.08 \pm 4.97$ & $3.45 \pm 2.48$ & $2.33 \pm 2.01$ \\
\hline BAL eosinophils $\times 10^{6} \cdot \mathrm{L}^{-1}$ & $1.04 \pm 0.940$ & $0.43 \pm 0.33$ & $4.88 \pm 4.42$ & $3.00 \pm 3.67$ & $6.79 \pm 14.3$ & $1.70 \pm 1.39$ & $0.0 \pm 0.0$ & $0.420 \pm 0.380$ \\
\hline BALF S-Alb $g \cdot \mathrm{L}^{-1}$ & $40.4 \pm 1.95$ & $39.7 \pm 2.08$ & $39.6 \pm 1.85$ & $38.7 \pm 2.24$ & $37.7 \pm 1.33$ & $39.0 \pm 2.72$ & $37.7 \pm 1.71$ & $40.1 \pm 1.95$ \\
\hline AB-PAS positive cells $\%$ & $13.8 \pm 12.2$ & $15.4 \pm 14.9$ & $22.4 \pm 16.8$ & $18.8 \pm 12.7$ & $23.2 \pm 16.1$ & $27.0 \pm 16.1$ & $26.3 \pm 14.9$ & $35.0 \pm 6.3$ \\
\hline Blood platelets $\times 10^{9} \cdot \mathrm{L}^{-1}$ & $214 \pm 33.7$ & $276 \pm 36.8$ & $244 \pm 46.4$ & $296 \pm 65.4$ & $279 \pm 65.9$ & $299 \pm 55.0$ & $255 \pm 54.1$ & $257 \pm 64.9$ \\
\hline Blood leukocytes $\times 10^{9} \cdot \mathrm{L}^{-1}$ & $5.57 \pm 1.18$ & $5.44 \pm 0.858$ & $7.87 \pm 1.38$ & $7.10 \pm 1.86$ & $7.31 \pm 1.44$ & $8.25 \pm 2.56$ & $6.00 \pm 1.04$ & $7.80 \pm 2.48$ \\
\hline
\end{tabular}

Data are presented as mean \pm SD, unless otherwise stated. Healthy: healthy never-smokers; smokers: healthy smokers; COPD: chronic obstructive pulmonary disease smokers; COPD ExS: chronic obstructive pulmonary disease ex-smokers; GOLD: Global Initiative for Chronic Obstructive Lung Disease; BMI: body mass index; CRP: C-reactive protein; BAL: bronchoalveolar lavage; FEV1: forced expiratory volume in $1 \mathrm{~s}$; VC: vital capacity; FVC: forced vital capacity; BALF: BAL fluid; S-Alb: serum albumin; AB-PAS: alcian blue-periodic acid-Schiff. NA: not applicable.

table E2 and table E3, respectively. Isoprostanes were screened via LC-MS/MS as previously reported [24]. Cysteinyl leukotrienes were measured by immunoassay as described in the online supplement.

\section{mRNA analysis}

mRNA was isolated from BAL cells from a subset of the COSMIC cohort based upon sample availability ( $n=6$ female smokers with COPD, $n=20$ female smokers with normal lung function). The distribution of the female smokers and COPD patients subjected to mRNA analysis was analysed by principal component analysis (PCA) based upon their full clinical profile. The corresponding scores plot demonstrated that the six samples analysed were representative of the population (data not shown). mRNA was subjected to low input quick amplification, and Cy3-CTP single-color labelling (Agilent Technologies, Santa Clara, CA, USA) and hybridised to Agilent human whole-genome arrays containing 41000 probes corresponding to 19596 genes. Microarray datasets were normalised using quantile normalisation with limma in Bioconductor (https://bioconductor.org). Statistical analyses were performed on probe intensities from a selected subset of 13 probes representing seven genes (EPHX1, EPHX2, CYP2C, CYP1A2, CYP2J, CYP3A4, and CYP2E1) involved in the biosynthesis of the EpOMEs and DiHOMEs.

\section{Statistical analysis}

Statistical analyses were performed by comparing smoking and nonsmoking individuals separately in order to limit the confounding effects of smoking. Univariate statistical analysis was performed by non-parametric Mann-Whitney test $(\mathrm{p}<0.05)$. False discovery rate estimations were performed by calculating the Storey q-values using the package "qvalue" from Bioconductor in R 3.2.2. Multivariate statistical modelling was performed using SIMCA version 13.0 (Umetrics, Umeå, Sweden) employing a combination of PCA and orthogonal projections to latent structures (OPLS) models. Only variables detected in $\geqslant 75 \%$ of the subjects in at least one patient group were included. Analytes present at levels 

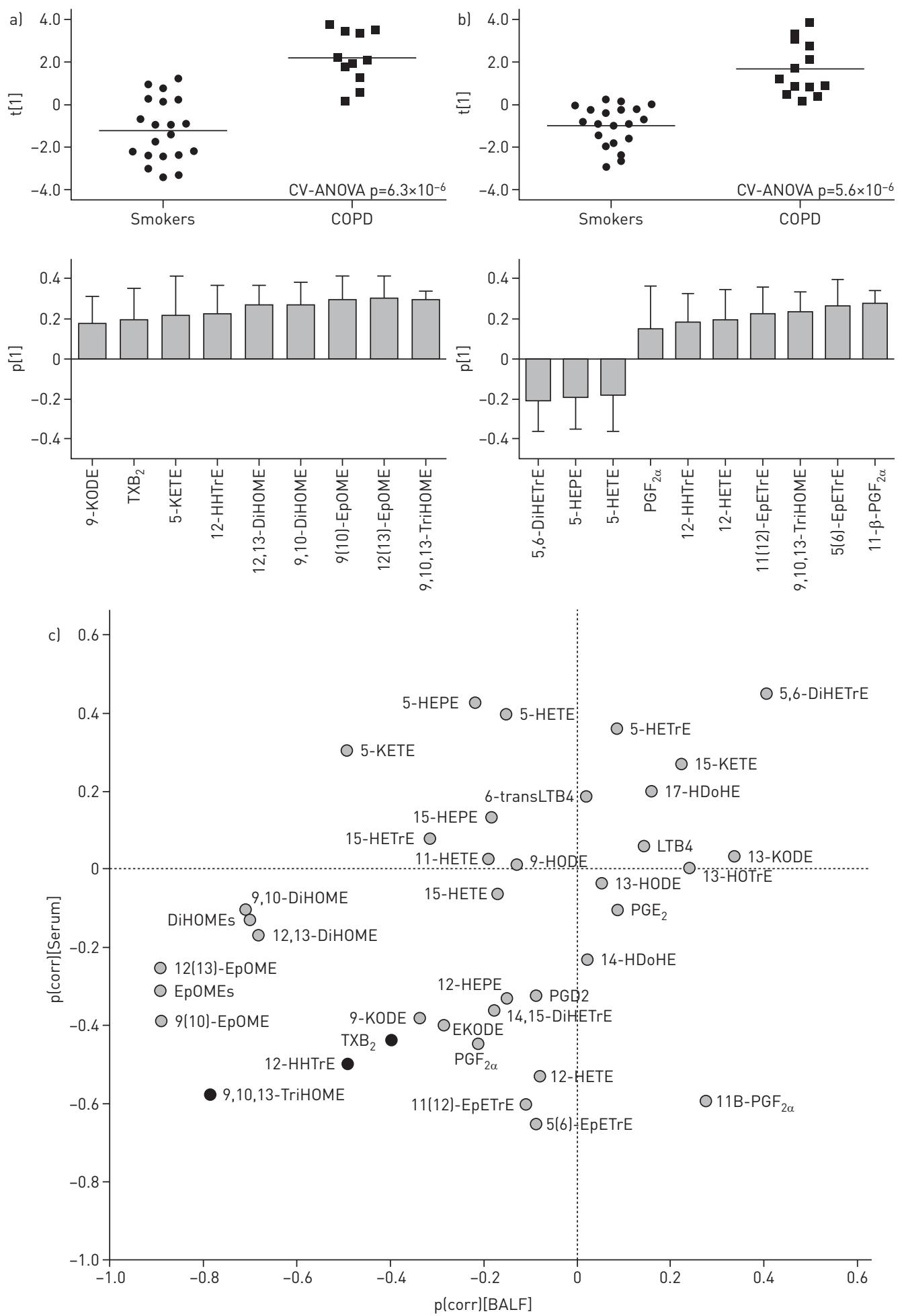

FIGURE 2 Optimised orthogonal projections to latent structures (OPLS) models for female smokers with normal lung function versus chronic obstructive pulmonary disease (COPD) patients. a) Bronchoalveolar lavage fluid (BALF) and b) serum. c) Shared and unique structures (SUS) plot for lipid mediators in BALF ( $x$-axis) versus serum (y-axis) for females. In the OPLS models (a and b), upper panels display score plots with one predictive component (t[1]), and lower panels display loadings ( $p[1]$ ). Smokers with normal lung function (smokers, closed circles), COPD smokers (COPD, closed squares). The corresponding models for the male comparisons were not significant in $\operatorname{BALF}(p=1.0)$, but did reach significance in serum ( $p=0.03)$. Lipid mediator nomenclature is provided in table E1 of the supplementary material. 
below the limit of detection were set to $25 \%$ of limit of detection for multivariate model construction. Data were $\log$ transformed, mean centered and scaled to unit variance. Model statistics are reported by the cumulative correlation coefficient $\left(R^{2} Y\right)$, the predictive variance based on seven-fold cross-validation $\left(Q^{2}\right)$, and cross-validated ANOVA p-values for OPLS models. PCA of lipid mediator levels was performed for data quality assessment in BALF and serum (data not shown). Variable selection for OPLS model optimisation was performed iteratively based on the variable influence in projection (VIP) and scaled loadings of the predictive component ( $\mathrm{p}[\mathrm{corr}])$ as previously described $[18,25]$. Comparison of two OPLS models was performed using shared and unique structure (SUS) analysis. Correlations between subsets of lipid mediators and clinical variables were evaluated using PLS (SIMCA), reported as the $r$ and p-value of the inner relation.

\section{Results}

\section{Cohort description}

The clinical characteristics of the subjects are provided in table 1. A total of 94 lipid mediators were screened in BALF using three different analytical methods, with 40 fulfilling the criterion of detection in $\geqslant 75 \%$ of the subjects in at least one group (table E4). In serum, 86 lipid mediators were screened (cysteinyl leukotrienes and isoprostanes were not screened in serum), of which 70 fulfilled the detection criterion (table E5). To minimise the confounding effects of smoking in COPD, the smoking population (smokers with normal lung function ("smoker") and smoking COPD patients ("COPD")) was analysed separately from the non-smoking populations (healthy never-smoker subjects ("healthy") and ex-smoker COPD patients (“COPD ExS")). No gender differences were observed in the quantified lipid mediators for any of the group comparisons in either BALF or serum ( $q>0.9$, data not shown). The concentrations of BAL immune cells (macrophages, neutrophils, and eosinophils) and blood leukocytes were elevated with smoking (table 1), but not with COPD diagnosis (figure E2). The corresponding decrease in cell counts with smoking cessation in COPD patients was only significant for macrophages and eosinophils (table 1). No gender differences were observed. Blood platelets were higher in females versus males in the healthy smoking group, but not with COPD diagnosis (figure E2D).

\section{Multivariate modelling of COPD-related lipid mediators}

Supervised multivariate modelling was performed by OPLS analysis of lipid mediator levels in BALF. The initial joint gender model of smokers versus COPD resulted in a significant group separation $\left(\mathrm{R}^{2} \mathrm{Y}=0.47\right.$, $\left.\mathrm{Q}^{2}=0.33, \mathrm{p}=6 \times 10^{-5}\right)$. However, investigating each gender separately revealed that the group separation was driven by the female population. The OPLS model of female Smokers versus COPD was optimised via variable selection (figure E3) to give a significant robust group separation $\left(R^{2} Y=0.59, Q^{2}=0.57, p=6 \times 10^{-6}\right.$; figure 2a), while the corresponding analysis on males showed no differences $(p=1.0)$. The optimised model consisted of nine lipid mediators: 9,10,13-TriHOME (9,10,13-trihydroxy-11E-octadecenoic acid), 12(13)-EpOME (12[13] epoxy-9Z-octadecenoic acid), 9(10)-EpOME (9[10]-epoxy-12Z-octadecenoic acid), 9,10-DiHOME (9[10]-dihydroxy-12Z-octadecenoic acid), 12,13-DiHOME (12[13]-dihydroxy-12Zoctadecenoic acid), 12-HHTrE (12-hydroxy-5Z,8E,10E-heptadecatrienoic acid), 5-KETE (5-oxo-ETE, 5-oxo$6 E, 8 Z, 11 Z, 14 Z$-eicosatetraenoic acid), $\mathrm{TXB}_{2}$ (thromboxane $\mathrm{B}_{2}$ ) and 9-KODE (9-oxo-10E,12Z-octadecadienoic acid) (see table E1 for lipid mediator nomenclature). Subjecting the nine-lipid model to permutation tests confirmed that the observed differences did not occur by random (Y-intercept [500 permutations]: $\mathrm{R}^{2} \mathrm{Y}=0.05$, $\mathrm{Q}^{2}=-0.24$, figure E4). In addition, no effect of menopause status or oestrogen replacement therapy was observed on the specificity of the nine-lipid panel for female smokers with COPD (figure E5).

In serum, the optimised OPLS model comparing female smokers versus COPD yielded a significant group separation $\left(\mathrm{p}=6 \times 10^{-6}, \mathrm{R}^{2} \mathrm{Y}=0.61, \mathrm{Q}^{2}=0.56\right.$; figure $\left.2 \mathrm{~b}\right)$. The corresponding male OPLS model also gave a significant group separation ( $\mathrm{p}=0.03$, data not shown). Both gender models were driven by lower serum-abundances of 5-LOX products and increases in CYP-derived (5[6]-EpETrE, 11[12]-EpETrE) and putative platelet-derived products (12-HETE, 12-HHTrE) in COPD patients (females; figure 2b), and no gender-specific differences were observed by SUS analysis (figure E6).

\section{Univariate analysis of COPD-related lipid mediators}

Because the OPLS multivariate model in BALF evidenced strong gender-specificity, it was investigated in greater detail. The members of the 9-lipid panel from figure 2a were examined on an individual basis by univariate statistics (table E6). Correlation analyses were performed between each of the nine lipid mediators with age and pack-years. None of the lipids evidenced a significant correlation, except for 5-KETE, which correlated with pack-years in females $(\mathrm{r}=0.42, \mathrm{p}=0.02)$. In both genders, levels of 9,10,13-TriHOME were lower in smokers and then elevated with COPD diagnosis, although the significance of the shifts was greater in females (figure 3a). The 9-KODE levels (figure 3b), 5-KETE levels (figure 3c) as 
well as $\mathrm{TXB}_{2}$ and 12-HHTrE (figure 3d) were significantly greater in female smoking COPD patients compared to non-symptomatic smokers, with no alterations in the corresponding male populations.

The CYP-derived products of linoleic acid evidenced multiple shifts with smoking and COPD (figure 4). Both the EpOMEs (figure 4a) and DiHOMEs (figure 4b) were higher in female COPD patients relative to smokers. Of particular interest is that no differences were observed for the corresponding male populations. For comparison purposes, levels of the CYP-derived products of arachidonic acid, epoxy-eicosatrienoic acids (EpETrEs) and dihydroxy-eicosatrienoic acids (DiHETrEs), were only altered due to smoking, with no alterations between COPD versus smokers (figure E7). Accordingly, the gender specificity of the shifts was only associated with the linoleic acid pathway.

The relative level of epoxide formation from linoleic acid was estimated by the sum of EpOMEs and DiHOMEs (figure 4c). The observed trends followed those of EpOMEs: downregulation of linoleic acid epoxide formation in female smokers and higher levels in female COPD patients. In order to estimate the conversion of EpOMEs to DiHOMEs (inferred sEH activity), the ratio DiHOMEs/(EpOMEs+DiHOMEs) in each individual was determined (figure $4 \mathrm{~d}$ ). As expected, the ratio reflected the gender differences observed in the CYP-derived linoleates, although this comparison cannot distinguish increases in substrate availability from differences in the enzymatic activity of sEH.

\section{COPD classification model was validated in an independent cohort}

The nine-lipid panel from figure 2a was quantified in BALF from an independent cohort of smokers and COPD patients from both genders. Using the OPLS classification model developed for the initial cohort (figure $2 \mathrm{a}$ ), the females in the validation cohort were classified with an accuracy of $92.3 \%(\mathrm{p}=0.005)$. Of the 13 females (seven smokers, six COPD), all were correctly classified except for one COPD patient, who was incorrectly classified as a smoker (table E7), resulting in $83.3 \%$ sensitivity and $100 \%$ specificity. Given that there was no OPLS model for the corresponding male population, the female model from figure $2 \mathrm{a}$ was used to perform an equivalent analysis with the males in the validation cohort. The males (nine smokers, five COPD) were classified with an accuracy of $57.1 \%$ ( $\mathrm{p}=0.5$, table E8).
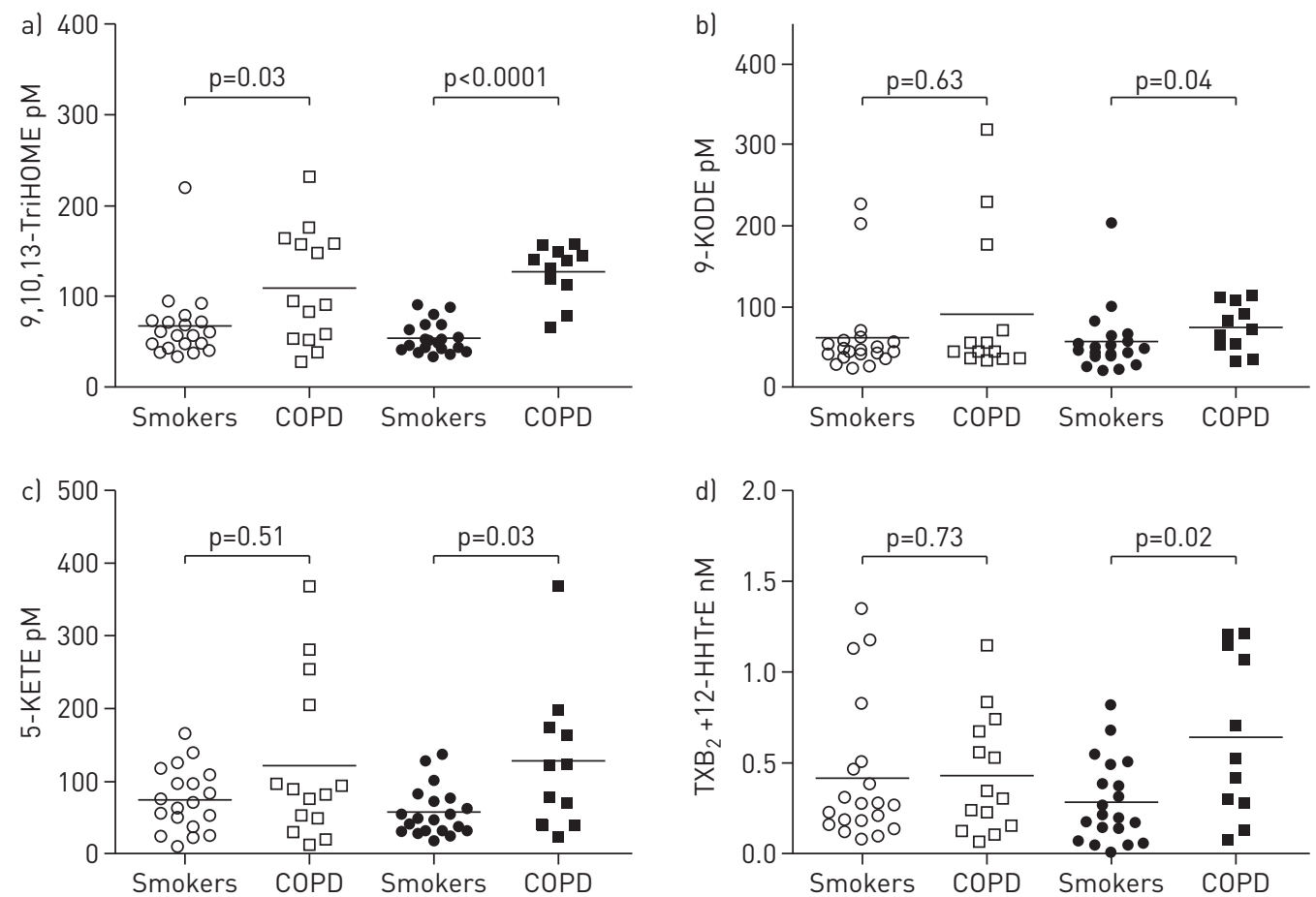

FIGURE 3 Lipid mediator levels in bronchoalveolar lavage fluid (BALF) in relation to sex, smoking status and chronic obstructive pulmonary disease (COPD). a) 9,10,13-TriHOME, b) 9-KODE. c) 5-KETE and d) sum of TXB and $12-\mathrm{HHTrE}$ (TXA 2 synthase pathway). Subjects are divided into smokers with normal lung function (smokers, circles) and smokers with COPD (COPD, squares). Open symbols indicate males and closed symbols females. Significance is indicated by the non-parametric Mann-Whitney test. Lipid mediator nomenclature is as follows: 9,10,13-TriHOME (9,10,13-trihydroxy-11E-octadecenoic acid), 9-KODE (9-oxo-10E,12Z-octadecadienoic acid), 5-KETE (5-oxo-ETE, 5-oxo-6E,8Z,11Z,14Z-eicosatetraenoic acid), 12-HHTrE (12-hydroxy-5Z,8E, 10E-heptadecatrienoic acid), and $\mathrm{TXB}_{2}$ (thromboxane $\mathrm{B}_{2}$ ). 

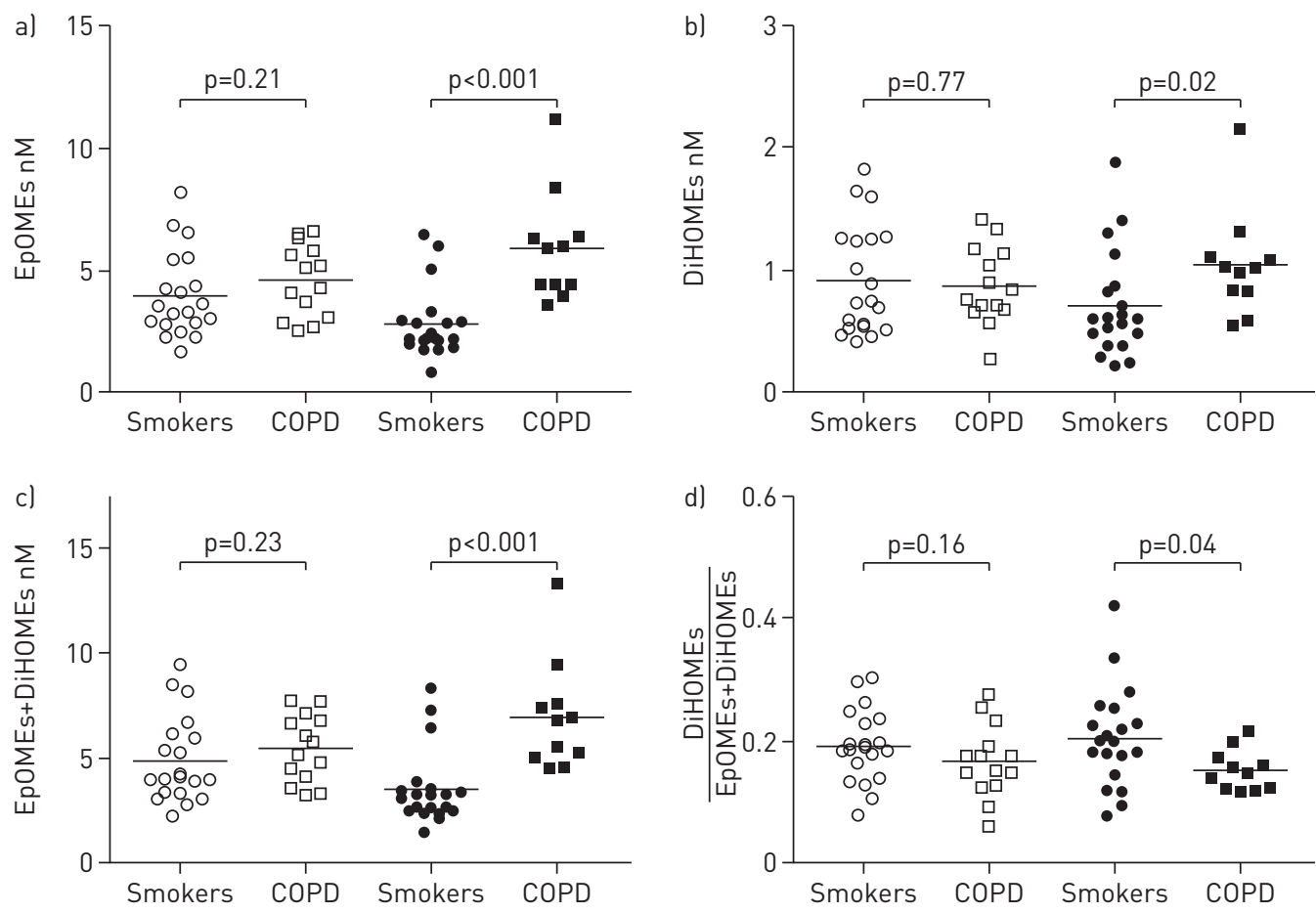

FIGURE 4 Cytochrome P450-derived linoleate mediators in bronchoalveolar lavage fluid (BALF) in relation to gender, smoking status and disease. a) EpOMEs, b) DiHOMEs, c) EpOMEs+DiHOMEs and d) DiHOMEs/(EpOMEs +DiHOMEs). Subjects are divided into smokers with normal lung function (Smokers, circles) and smokers with COPD (COPD, squares). Open symbols indicate males and closed symbols females. Significance is indicated by the non-parametric Mann-Whitney test. Lipid mediator nomenclature is as follows: EpOMEs are 9(10)-EpOME (9[10]-epoxy-12Z-octadecenoic acid) and 12(13)-EpOME (12[13]epoxy-9Z-octadecenoic acid); and DiHOMEs are 9,10-DiHOME (9[10]-dihydroxy-12Z-octadecenoic acid) and 12,13-DiHOME (12[13]-dihydroxy-12Z-octadecenoic acid).

\section{Linoleic acid-derived species correlate with lung function and mRNA levels in female COPD patients}

The EpOME and DiHOME lipid mediators driving the separation between female smokers and COPD patients correlated stronger with lung function (FEV1 and FEV1/FVC) in female COPD patients (PLS inner relation $\mathrm{r}=0.87, \mathrm{p}=9 \times 10^{-4}$, figure $5 \mathrm{a}$ ), than in female smokers (PLS inner relation $\mathrm{r}=0.50, \mathrm{p}=0.02$, data not shown). The EPOME and DiHOME lipid profiles of the female COPD patients also correlated significantly with the individual lung function variables FEV1 (PLS Inner relation $\mathrm{r}=0.69, \mathrm{p}=0.02$ ) and FEV1/FVC (PLS inner relation $\mathrm{r}=0.76, \mathrm{p}=0.01$ ).

The mRNA for genes putatively associated with the biosynthesis of the EpOMEs and DiHOMEs was identified based upon known biochemical pathways. The gene assignments were as follows: relevant CYPs for EpOMEs based upon the KEGG linoleic acid pathway (hsa00591: CYP1A2, CYP2C, CYP2J, CYP3A4 and CYP2E1), and two epoxide hydrolase (EH) genes for the DiHOMEs (EPHX1, EPHX2). The corresponding correlation between FEV1 and mRNA levels was stronger for female COPD patients $(\mathrm{r}=0.96, \mathrm{p}=0.003$, figure $5 \mathrm{~b})$ than for female smokers $(\mathrm{r}=0.66, \mathrm{p}=0.03$, data not shown). No relationships with lung function or mRNA levels were observed for the corresponding male populations.

\section{Linoleic acid-derived species correlate with goblet cell abundance in female COPD patients}

To obtain insight into the cellular origin of the nine-lipid panel, the abundance of the BAL immune cells (macrophages, lymphocytes, neutrophils, eosinophils) as well as structural cells from bronchial brushings (goblet cells) and blood platelets were correlated with the lipids on a pathway-specific basis. In the female COPD group, 9,10,13-TriHOME levels correlated with neutrophil abundance ( $\mathrm{r}=0.77, \mathrm{p}=0.005$; figure $5 \mathrm{c}$ ), while the remaining five linoleates (DiHOMEs, EpOMEs and 9-KODE) displayed a weaker correlation with eosinophil abundance $(\mathrm{r}=0.67, \mathrm{p}=0.02)$. No significant correlations were found for the corresponding male groups (figure E8). Both female and male COPD patients exhibited a weak correlation between blood platelet levels and the nine-lipid panel $(\mathrm{r}=0.64, \mathrm{p}=0.047 ; \mathrm{r}=0.66, \mathrm{p}=0.03$, respectively; data not shown). No correlations were observed for blood platelets and any of the individual compounds from the nine-lipid panel. All nine lipids correlated with goblet cells in female COPD patients ( $r=0.84, p=0.005)$, with the correlation driven primarily by the EpOMEs $(r=0.72, p=0.03$; figure $5 \mathrm{~d})$. The corresponding correlation in 

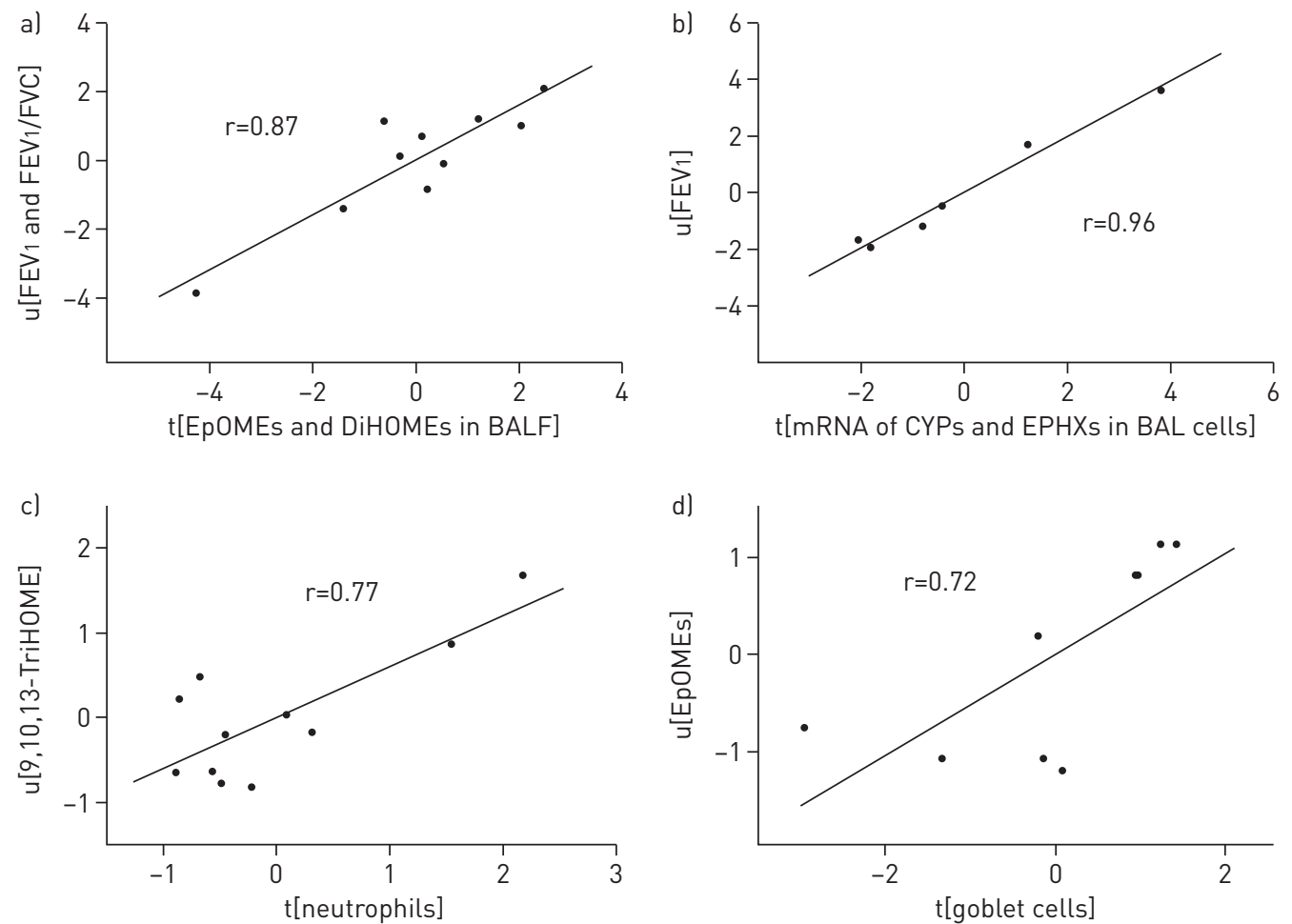

FIGURE 5 Correlation between lipid mediators in bronchoalveolar lavage fluid (BALF) with lung function, mRNA, neutrophils and goblet cells. a) The correlation between lung function parameters (forced expiratory volume in $1 \mathrm{~s}(\mathrm{FEV} 1)$ and $\mathrm{FEV} /$ /forced expiratory volume (FVC)) versus the concentrations of EpOMEs and DiHOMEs in female COPD smokers (PLS inner relation, $r=0.87, p=0.0009, n=10$ ). b) The correlation of FEV1 versus mRNA related to the EPOME and DiHOME lipid mediators (EPHX1, EPHX2, CYP1A2, CYP2C, CYP2J, CYP3A4 and CYP2E1) of a subset of the female COPD smokers group (PLS inner relation, $r=0.96, p=0.003, n=6$ ). c) The correlation between neutrophil abundance in BALF and 9,10,13-TriHOME (PLS inner relation, $r=0.77, p=0.005$, $\mathrm{n}=11$ ). d) The correlation between goblet cell abundance versus the 2 EpOME lipid mediators (leukotoxin and isoleukotoxin; PLS inner relation, $r=0.72, p=0.03, n=9)$. Lipid mediator nomenclature is as follows: EpOMEs=9(10)EpOME (9[10]-epoxy-12Z-octadecenoic acid) and 12(13)-EpOME (12[13]epoxy-9Z-octadecenoic acid); DiHOMEs=9,10-DiHOME (9[10]-dihydroxy-12Z-octadecenoic acid) and 12,13-DiHOME (12[13]-dihydroxy-12Z-octadecenoic acid); 9,10,13-TriHOME $(9,10,13$-trihydroxy-11E-octadecenoic acid).

male COPD patients was weaker for the nine-lipid panel ( $\mathrm{r}=0.65, \mathrm{p}=0.02$; data not shown) and non-significant for the EpOMEs ( $r=0.46, p=0.14$, figure $\mathrm{E} 8 \mathrm{C})$.

\section{Discussion}

COPD is a heterogeneous inflammatory disease that is a leading cause of morbidity and mortality worldwide. This heterogeneity may manifest in patient sub-phenotypes involving different molecular pathways in onset and development of the disease. Results from the current study identified increases in lipid mediators from the linoleic acid-derived CYP pathway, as well as arachidonic acid-derived products of thromboxane synthase and 5-LOX in smokers with early-stage COPD compared with smokers with normal lung function. These differences were driven by the female population, with a nine-lipid panel classifying female smokers with COPD from female smokers with normal lung function $\left(p=6.3 \times 10^{-6}\right.$; figure $\left.2 a\right)$. While the classification model for women was robust, and confirmed with $92.3 \%$ accuracy in an independent validation cohort, no differences were observed in the corresponding male populations $(p=1.0)$. In contrast, no gender-based differences were observed for the corresponding smokers with normal lung function or healthy never-smokers groups, indicating that the observed gender-related differences were associated with COPD disease phenotype rather than generic gender-differences. As such, the putative pattern identified in this study is applicable to a female-dominated disease sub-phenotype, thereby corresponding to our previously reported alterations in the BAL cell proteome of the same cohort [18].

The strongest drivers of the gender-specificity were the linoleates in BALF (the EpOMEs (leukotoxin and isoleukotoxin) and DiHOMEs (leukotoxin diol and isoleukotoxin diol) as well as 9,10,13-TriHOME). The EpOMEs are epoxides that can be produced enzymatically from linoleic acid via CYP activity, which are further converted to the corresponding 1,2-diols, DiHOMEs, via sEH [26] (figure 1). EpOMEs and DiHOMEs have been studied in models of respiratory diseases $[16,27,28]$, especially in rodent models of 
ARDS [14, 29], with the DiHOMEs demonstrated to exert cytotoxicity [17, 30]. The DiHOMEs were reported to decrease net ion flux in alveolar epithelial monolayers and increase intercellular junction permeability [17, 30], both of which are known contributing factors to chronic bronchitis [31]. Chronic bronchitis has, with some contradictions [32], been reported to be a more prevalent clinical manifestation among female COPD patients by many investigators [4, 33, 34], including twin studies [35]. BALF EpOME levels in female COPD patients correlated with goblet cell abundance, which have been reported to increase with smoking [36] and possess CYP activity [37]. Accordingly, these findings suggest a mechanism, by which goblet cell hyperplasia in female smokers leads to elevated production of EpOMEs and the commensurate DiHOMEs, which potentiates the onset of chronic bronchitis and COPD. These findings are of particular clinical relevance due to the interest in targeting the sEH pathway for COPD treatment. For example, GlaxoSmithKline has conducted two Phase I clinical trials to evaluate the use of sEH inhibitors for the treatment of COPD (NCT01762774 and NCT02006537).

Little is known about the 9,10,13-TriHOME, with its route of formation and mechanism of action unclear. TriHOMEs have been reported to exert prostaglandin $\mathrm{E}_{2}$-like activity of relaxing vascular smooth muscle cells in vitro [38] and to be altered in asthma [39]. Levels of the 9,10,13-TriHOME correlated with neutrophils (figure 5c), which possess 9,10,13-TriHOME synthesis activity [40]. Accordingly, the 9,10,13-TriHOME is likely formed in the lung via a neutrophil LOX-dependent process, followed by sEH activity. The biosynthetic route and function of the 9-KODE are equally unclear, although the linoleic acid oxidative metabolites including the monohydroxy- and keto-octadecadienoic acid are implicated in a variety of pathologies $[41,42]$ as well as reported to exert peroxisome proliferator-activated receptor- $\gamma$ agonist activity [43].

Other primary differences between female smokers and COPD patients include production of the arachidonic acid-derived $\mathrm{TXB}_{2}, 12$-HHT and 5-KETE. The thromboxane synthase products 12-HHT and $\mathrm{TXB}_{2}$ are classic platelet-derived lipids [44], thereby suggesting a role of platelet activation in COPD. These two lipids as well as 9,10,13-TriHOME were the only species to correlate between female BALF and serum models (SUS plot, figure 2c), indicating a systemic relationship for these three mediators. Furthermore, the BALF levels of the full nine-lipid panel correlated with blood platelets for smoking COPD patients in both sexes. Platelet-neutrophil transcellular interactions have been recently shown to regulate lung inflammation via the production of lipid mediators [45], further suggesting that platelets may play a role in the inflammatory-derived pathobiology of lung disease. 5-KETE is a potent chemoattractant for eosinophils [46] that can be synthesised by airway epithelial cells [47], neutrophils [48] and blood platelets [49] in response to oxidative stress. Levels of 5-KETE have been postulated to prolong pulmonary inflammation and potentially contribute to conditions such as severe asthma [46].

Mechanistically, it is unclear what is driving the observed gender-specific shift. Linoleic acid is the major dietary fat and cannot be de novo synthesised by mammals [50]. Its metabolites are known to be biologically active, with the DiHOMEs generally considered to be pro-inflammatory and the EpOMEs anti-inflammatory [51]. A meta-analysis found no gender differences in the levels of linoleic acid in plasma lipids [52], suggesting that the observed differences are not associated with gender-related dietary intake. Higher concentrations of EpOMEs and the corresponding DiHOMEs in BALF of females with COPD suggest a gender-specific upregulation of the CYP-pathway, which is related to smoking-driven goblet cell hyperplasia. Gender specificity in the expression of CYP enzymes has been extensively reported in rodent tissues [53], although less is known about the patterns in humans, especially the lung [54]. Accordingly, it would be of interest to investigate the gender-dependent expression and activity of CYP enzymes in goblet cells and their role in the onset and progression of COPD.

These findings also support recent reports of platelet activation in COPD. Platelets have been proposed as key mediators in inflammatory lung disease [55], playing a critical role in the recruitment of neutrophils, eosinophils and lymphocytes [56]. The production of platelet-activation factor (PAF) has been reported to increase in response to cigarette smoke [57]. Patients with stable COPD have increased circulating platelet-monocyte aggregates, and PAF increases during an acute exacerbation [58]. Anti-platelet therapy (i.e. clopidogrel) [59] as well as statin therapy [60] (which reduces platelet activation) have been suggested in the treatment of COPD. Given that several components of the nine-lipid panel identified in this study can be linked to platelet activation, these findings further support the theory of platelet involvement in the pathobiology of COPD.

\section{Conclusions}

Female gender has been suggested as a risk factor in susceptibility to the lung-damaging effects of cigarette smoke, and both smokers and nonsmokers with COPD are more likely to be female. In the current study, a nine-lipid panel evidenced specificity in COPD. The identified lipid mediators included compounds previously suggested to be of importance in disease including 5-KETE and thromboxane synthase 
products $\left(\mathrm{TXB}_{2}\right.$ and 12-HHT), as well as more novel lipids produced via the CYP pathway from linoleic acid. These results suggest a gender-based phenotypic difference in the production of lipid mediators associated with inflammatory disease. The female-specific correlation between goblet cells and EpOME levels in BALF in combination with their purported role in decreased ion flux in airway epithelial monolayers and increased intercellular junction permeability suggests a mechanism by which increased production of EpOMEs could lead to more frequent clinical manifestations of chronic bronchitis in the female COPD population. In combination with the reported association between linoleic acid-derived mediators with multiple organ failure and ARDS, it is possible that this pathway plays an important role in the aetiology of certain COPD sub-phenotypes.

\section{Acknowledgements}

We thank the research nurses Heléne Blomqvist, Margitha Dahl and Gunnel de Forest (Lung-Allergy Clinic, Karolinska University Hospital Solna, Stockholm, Sweden) and Ann-Sofie Lantz (Department of Respiratory Medicine and Allergy, Karolinska University Hospital Huddinge, Stockholm, Sweden) as well as biomedical analyst Benita Engvall (Respiratory Medicine Unit, Department of Medicine Solna, Karolinska Institute, Stockholm, Sweden) for sample collection and preparation, as well as all of the volunteers for making the study possible. We also thank Ingrid Delin and Johan Bood (Lung and Airway Research Unit, Institute of Environmental Medicine, Karolinska Institutet, Stockholm, Sweden) for performing the CysLT analyses.

\section{References}

1 Mannino DM, Buist AS. Global burden of COPD: risk factors, prevalence, and future trends. Lancet 2007; 370: $765-773$.

2 Pauwels RA, Rabe KF. Burden and clinical features of chronic obstructive pulmonary disease (COPD). Lancet 2004; 364: 613-620.

3 Ohar J, Fromer L, Donohue JF. Reconsidering sex-based stereotypes of COPD. Prim Care Respir J 2011; 20: 370-378.

4 Han MK, Postma D, Mannino DM, et al. Gender and chronic obstructive pulmonary disease: why it matters. Am J Respir Crit Care Med 2007; 176: 1179-1184.

5 Camp PG, Coxson HO, Levy RD, et al. Sex differences in emphysema and airway disease in smokers. Chest 2009; 136: $1480-1488$.

6 Sorheim IC, Johannessen A, Gulsvik A, et al. Gender differences in COPD: are women more susceptible to smoking effects than men? Thorax 2010; 65: 480-485.

7 Gan WQ, Man SP, Postma DS, et al. Female smokers beyond the perimenopausal period are at increased risk of chronic obstructive pulmonary disease: a systematic review and meta-analysis. Respir Res 2006; 7: 52.

8 Barnes PJ. Mediators of chronic obstructive pulmonary disease. Pharmacol Rev 2004; 56: 515-548.

9 Pinkerton KE, Harbaugh M, Han MK, et al. Women and Lung Disease. Sex Differences and Global Health Disparities. Am J Respir Crit Care Med 2015; 192: 11-16.

10 Lundström SL, Balgoma D, Wheelock ÅM, et al. Lipid mediator profiling in pulmonary disease. Curr Pharm Biotechnol 2011; 12: 1026-1052.

11 Dagouassat M, Gagliolo JM, Chrusciel S, et al. The cyclooxygenase-2-prostaglandin $\mathrm{E}_{2}$ pathway maintains senescence of chronic obstructive pulmonary disease fibroblasts. Am J Respir Crit Care Med 2013; 187: 703-714.

12 Bozinovski S, Uddin M, Vlahos R, et al. Serum amyloid A opposes lipoxin $\mathrm{A}_{4}$ to mediate glucocorticoid refractory lung inflammation in chronic obstructive pulmonary disease. Proc Natl Acad Sci USA 2012; 109: 935-940.

13 Snelgrove RJ, Jackson PL, Hardison MT, et al. A critical role for LTA4H in limiting chronic pulmonary neutrophilic inflammation. Science 2010; 330: 90-94.

14 Zheng J, Plopper CG, Lakritz J, et al. Leukotoxin-diol: A putative toxic mediator involved in acute respiratory distress syndrome. Am J Respir Cell Mol Biol 2001; 25: 434-438.

15 Ozawa T, Sugiyama S, Hayakawa M, et al. Existence of leukotoxin 9,10-epoxy-12-octadecenoate in lung lavages from rats breathing pure oxygen and from patients with the adult respiratory distress syndrome. Am Rev Respir Dis 1988; 137: 535-540.

16 Ishizaki T, Ozawa T, Voelkel NF. Leukotoxins and the lung. Pulm Pharmacol Ther 1999; 12: 145-155

17 Moghaddam MF, Grant DF, Cheek JM, et al. Bioactivation of leukotoxins to their toxic diols by epoxide hydrolase. Nat Med 1997; 3: 562-566.

18 Kohler M, Sandberg A, Kjellqvist S, et al. Gender differences in the bronchoalveolar lavage cell proteome of patients with chronic obstructive pulmonary disease. J Allergy Clin Immunol 2013; 131: 743-751.

19 Mikko M, Forsslund H, Cui L, et al. Increased intraepithelial (CD103+) CD8+ T cells in the airways of smokers with and without chronic obstructive pulmonary disease. Immunobiology 2013; 218: 225-231.

20 Forsslund H, Mikko M, Karimi R, et al. Distribution of T-cell subsets in BAL fluid of patients with mild to moderate COPD depends on current smoking status and not airway obstruction. Chest 2014; 145: 711-722.

21 Karimi R, Tornling G, Forsslund H, et al. Lung density on high resolution computer tomography (HRCT) reflects degree of inflammation in smokers. Respir Res 2014; 15: 23.

22 Blidberg K, Palmberg L, James A, et al. Adhesion molecules in subjects with COPD and healthy non-smokers: a cross sectional parallel group study. Respir Res 2013; 14: 47.

23 Löfdahl JM, Cederlund K, Nathell L, et al. Bronchoalveolar lavage in COPD: fluid recovery correlates with the degree of emphysema. Eur Respir J 2005; 25: 275-281.

24 Balgoma D, Larsson J, Rokach J, et al. Quantification of lipid mediator metabolites in human urine from asthma patients by electrospray ionization mass spectrometry: controlling matrix effects. Anal Chem 2013; 85: 7866-7874.

25 Wheelock AM, Wheelock CE. Trials and tribulations of 'omics data analysis: assessing quality of SIMCA-based multivariate models using examples from pulmonary medicine. Mol Biosyst 2013; 9: 2589-2596.

26 Morisseau C, Hammock BD. Impact of soluble epoxide hydrolase and epoxyeicosanoids on human health. Annu Rev Pharmacol Toxicol 2013; 53: 37-58. 


\section{5; 23: 361-363.} acid metabolites contributes to inflammatory hyperalgesia. Proc Natl Acad Sci USA 2009; 106: 18820-18824. Struct Mol Biol 2008; 15: 924-931.

44 Weyrich AS, Zimmerman GA. Platelets in lung biology. Annu Rev Physiol 2013; 75: 569-591.

45 Abdulnour RE, Dalli J, Colby JK, et al. Maresin 1 biosynthesis during platelet-neutrophil interactions is organ-protective. Proc Natl Acad Scie USA 2014; 111: 16526-16531.

46 Powell WS, Rokach J. The eosinophil chemoattractant 5-oxo-ETE and the OXE receptor. Prog Lipid Res 2013; 52: 651-665.

47 Erlemann KR, Cossette C, Gravel S, et al. Airway epithelial cells synthesize the lipid mediator 5-oxo-ETE in response to oxidative stress. Free Radic Biol Med 2007; 42: 654-664.

48 Powell WS, Gravelle F, Gravel S. Phorbol myristate acetate stimulates the formation of 5-oxo-6,8,11,14eicosatetraenoic acid by human neutrophils by activating NADPH oxidase. J Biol Chem 1994; 269: 25373-25380.

49 Erlemann KR, Rokach J, Powell WS. Oxidative stress stimulates the synthesis of the eosinophil chemoattractant 5-oxo-6,8,11,14-eicosatetraenoic acid by inflammatory cells. J Biol Chem 2004; 279: 40376-40384.

50 Whelan J, Fritsche K. Linoleic acid. Adv Nutr 2013; 4: 311-312.

51 Newman JW, Morisseau C, Hammock BD. Epoxide hydrolases: their roles and interactions with lipid metabolism. Prog Lipid Res 2005; 44: 1-51.

52 Lohner S, Fekete K, Marosvolgyi T, et al. Gender differences in the long-chain polyunsaturated fatty acid status: systematic review of 51 publications. Ann Nutr Metab 2013; 62: 98-112.

53 Waxman DJ, Holloway MG. Sex differences in the expression of hepatic drug metabolizing enzymes. Mol Pharmacol 2009; 76: 215-228.

54 Ben-Zaken Cohen S, Pare PD, Man SF, et al. The growing burden of chronic obstructive pulmonary disease and lung cancer in women: examining sex differences in cigarette smoke metabolism. Am J Respir Crit Care Med 2007; 176: 113-120.

55 Kuebler WM. Selectins revisited: the emerging role of platelets in inflammatory lung disease. J Clin Invest 2006; 116: 3106-3108

56 Tabuchi A, Kuebler WM. Endothelium-platelet interactions in inflammatory lung disease. Vascul Pharmacol 2008; 49: $141-150$.

57 Sharma J, Young DM, Marentette JO, et al. Lung endothelial cell platelet-activating factor production and inflammatory cell adherence are increased in response to cigarette smoke component exposure. Am J Physiol Lung Cell Mol Physiol 2012; 302: L47-L55.

58 Harrison MT, Short P, Williamson PA, et al. Thrombocytosis is associated with increased short and long term mortality after exacerbation of chronic obstructive pulmonary disease: a role for antiplatelet therapy? Thorax 2014; 69: 609-615.

59 Maclay JD, McAllister DA, Johnston S, et al. Increased platelet activation in patients with stable and acute exacerbation of COPD. Thorax 2011; 66: 769-774.

60 Janda S, Park K, FitzGerald JM, et al. Statins in COPD: a systematic review. Chest 2009; 136: 734-743. 